MRC.PH.TH-6-96

hep-th/9604167

\title{
CONSISTENT INTERACTIONS IN TERMS OF THE GENERALIZED FIELDS METHOD
}

\author{
Ömer F. DAYI" \\ TUBITAK - Marmara Research Center, Research Institute for Basic Sciences, \\ Department of Physics, P.O. Box 21, 41470 Gebze-TURKEY
}

\begin{abstract}
The interactions which preserve the structure of the gauge interactions of the free theory are introduced in terms of the generalized fields method of solving the Batalin-Vilkovisky master equation. It is shown that by virtue of this method the solution of the descent equations resulting from the cohomological analysis is provided straightforwardly. The general scheme is illustrated by applying it to spin-1 gauge field in 3 and 4 dimensions, to free $B F$ theory in $2-\mathrm{d}$ and to the antisymmetric tensor field in any dimension. It is shown that it reproduces the results obtained by cohomological techniques.
\end{abstract}

\footnotetext{
${ }^{1}$ E-mail address: dayi@yunus.mam.tubitak.gov.tr
} 
In a local gauge theory if the number of the physical degrees of freedom is modified when the interactions are switched off, there may be some negative norm states which yield inconsistencies in the scattering theory where one uses the asymptotic states [1]. To avoid it one deals with the local interactions which preserve the structure of the gauge generators of the free theory. These are the consistent interactions[2]. By using the cohomological aspects of the Batalin-Vilkovisky (BV) method of quantization of gauge theories 3] one obtains the descent equations whose solution yields the consistent interactions [4]- 6 .

The generalized fields method is proven to be powerful in the BV quantization of a large class of gauge theories[[7]- 8]. It offers the proper solution of the (BV) master equation in a straightforward way.

We show that when one deals with a free gauge theory whose action can be written first order in the exterior derivative $d$ and bilinear in fields, the generalized fields method can be applied. As far as the properties of gauge generators are unaltered when interactions are introduced the generalized fields method should still be applicable. Hence, it can be utilized to obtain the consistent interactions. Moreover, solution of the related descent equations is a by-product in this scheme, i.e. this method provides the solution of the cohomology problem in a straightforward and compact manner. We do not deal with the consistent interactions neither non-local nor possessing derivatives and obtainable by field redefinitions.

The general scheme is illustrated by applying it to the spin-1 gauge field in $d=4$ and $d=3$, whose consistent interactions yield Yang-Mills and ChernSimons field theories. The consistent interactions of the free $B F$ theory in $d=2$ are shown to lead to the gauge action of the quadratic Lie algebras 9. In all of the examples the solutions of the descent equations are presented. When we deal with the antisymmetric tensor field in D-dimension the application of the method is shown to provide in a straightforward manner the solution of the cohomology problem obtained in [6].

Let us deal with a free gauge theory whose action can be written as

$$
\mathcal{A}_{0}=\int\left(B^{a} \wedge d A^{a}+\frac{1}{2} \alpha_{a b} B^{a} \wedge B^{b}\right)
$$

where $\alpha$ can be a vanishing or a constant matrix which will be suppressed in the following. It is invariant under the infinitesimal gauge transformations

$$
\delta_{\Lambda} A^{a}=d \Lambda^{a}, \delta_{\Lambda} B^{a}=0 .
$$


To quantize this theory in terms of the BV method, one introduces the ghost, ghost of ghost fields and the antifields (minimal sector), by inspecting the reducibility properties of the gauge transformations. One can attribute to each of the fields a total form degree defined as the sum of the differential form degree and the ghost number. To apply the generalized fields method one collects the original fields and the ones introduced for applying the BV method in the generalized fields $\tilde{A}^{a}, \tilde{B}^{a}$ whose total form degrees are the same with the differential form degrees of the original fields $A^{a}, B^{a}$. The action written in terms of the generalized fields

$$
S_{0} \equiv \int \tilde{\mathcal{L}}_{0}=\int\left(\tilde{B}^{a} d \tilde{A}^{a}+\frac{1}{2} \tilde{B}^{a} \tilde{B}^{a}\right)_{(D, 0)}
$$

(the first of the numbers in the parentheses is the differential form degree, the second is the ghost number and if there is only one number it denotes the total form degree) is the proper solution of the master equation

$$
\left(S_{0}, S_{0}\right) \equiv \frac{\delta_{r} S_{0}}{\delta \tilde{A}^{a}} \frac{\delta_{l} S_{0}}{\partial \tilde{B}^{a}}-\frac{\delta_{r} S_{0}}{\delta \tilde{B}^{a}} \frac{\delta_{l} S_{0}}{\partial \tilde{A}^{a}}=0 .
$$

To prove this observe that due to the gauge transformations (2), in $\tilde{B}$ there is not any field possessing positive ghost number so that $(\tilde{B} \tilde{B})_{(D, 0)}=B \wedge B$ and there is not $B^{\star}$ term in $(\tilde{B} d \tilde{A})_{(D, 0)}$.

BRST transformation of a functional is defined as

$$
\Omega_{0} F(\tilde{A}, \tilde{B})=\frac{\partial_{r} F}{\partial \tilde{A}^{a}} \Omega_{0} \tilde{A}^{a}+\frac{\partial_{r} F}{\partial \tilde{B}^{a}} \Omega_{0} \tilde{B}^{a}
$$

where

$$
\Omega_{0} \tilde{A}^{a}=-\frac{\partial_{l} S_{0}}{\partial \tilde{B}^{a}}, \Omega_{0} \tilde{B}^{a}=\frac{\partial_{l} S_{0}}{\partial \tilde{A}^{a}}
$$

In terms of the BRST charge $\Omega_{0}$ (4) can equivalently be formulated as

$$
\Omega_{0} \tilde{\mathcal{L}}_{0}+d \omega_{(D-1,1)}=0,
$$

where $\omega_{(D-1,1)}$ is a suitable $(D-1)$-form possessing ghost number one. Moreover, due to the fact that $\left(\Omega_{0}+d\right)^{2}=0$, there are the descent equations

$$
\Omega_{0} \omega_{(D-1,1)}+d \kappa_{(D-2,2)}=0, \Omega_{0} \kappa_{(D-2,2)}+d \lambda_{(D-3,3)}=0, \cdots
$$


with suitable $\kappa, \lambda, \cdots$, vanishing at a certain step.

Obviously $\Omega_{0}$ maps $\tilde{A} \rightarrow \tilde{d A}$ and $\tilde{B} \rightarrow \tilde{d B}$ except

$$
\Omega_{0} B^{\star a}=d A^{a}+B^{a} .
$$

However, there is not any $B^{\star}$ term in $\tilde{\mathcal{L}}_{0}$, so that, the BRST transformation of the free (7) theory can be generalized as

$$
\left(\Omega_{0}+d\right)\left(\tilde{B}^{a} d \tilde{A}^{a}\right)_{(D)}=0 .
$$

We dropped the $B^{a} \wedge B^{a}$ term which does not transform under $\Omega_{0}$. (9) written in components produces (8) with the identification $\omega_{(D-1,1)} \equiv\left(\tilde{B}^{a} d \tilde{A}^{a}\right)_{(D-1,1)}$, $\kappa_{(D-2,2)} \equiv\left(\tilde{B}^{a} d \tilde{A}^{a}\right)_{(D-2,2)}$ and so on. A compact notation similar to (9) is also presented in 10

The consistent interactions will be introduced as cubic or higher order in the generalized fields $\tilde{\Phi} \equiv(\tilde{A}, \tilde{B})$ :

$$
\begin{aligned}
S_{i n t} & \equiv \sum_{k=1, M=3} g_{M}^{k} S_{M k}=\int \sum_{M=3}\left(g_{M} \tilde{\Phi}^{M}+g_{M}^{2} \tilde{\Phi}^{M+1}+\cdots\right)_{(D, 0)} \\
& =\left(g_{3} \tilde{\Phi}^{3}+g_{3}^{2} \tilde{\Phi}^{4}+\cdots+g_{4} \tilde{\Phi}^{4}+g_{4}^{2} \tilde{\Phi}^{5}+\cdots\right)_{(D, 0)},
\end{aligned}
$$

such that

$$
\left(S_{0}+S_{i n t}, S_{0}+S_{i n t}\right)=0 .
$$

$g_{M}$ are independent coupling constants, hence, (11) leads to

$$
\begin{aligned}
\left(S_{0}, S_{0}\right) & =0, \\
\left(S_{0}, S_{M 1}\right) & =0, \\
2\left(S_{0}, S_{M 2}\right)+\left(S_{M 1}, S_{M 1}\right) & =0,
\end{aligned}
$$

(13) can equivalently be written in terms of the BRST charge $\Omega_{0}$ as

$$
\Omega_{0} \tilde{\Phi}_{(D, 0)}^{M}+d k_{(D-1,1)}=0,
$$


where $k$ is a suitable $(D-1)$-form possessing ghost number one. As it has already mentioned $\Omega_{0}$ maps $\tilde{A} \rightarrow \tilde{d A}$ and $\tilde{B} \rightarrow \tilde{d B}$ except $B^{\star}$. Let the $B^{\star}$ dependent terms in $S_{M 1}$ are

$$
\left(\tilde{\Phi}_{(D, 0)}^{M}\right)_{B^{\star}} \sim\left(B^{\star} \tilde{\Phi}^{M-1}+B^{\star 2} \tilde{\Phi}^{M-2}+\cdots+B^{\star M-1} \tilde{\Phi}\right)_{(D, 0)} .
$$

Thus in (15) the terms which does not possess an exterior derivative will be

$$
\Omega_{0} \tilde{\Phi}_{(D, 0)}^{M}+d k_{D-1,1} \sim B \tilde{\Phi}^{M-1}+2 B B^{\star} \tilde{\Phi}^{M-2}+\cdots+(M-1) B^{\star M-2} \tilde{\Phi}
$$

There are two possibilities $i$ ) (16) vanishes due to symmetry properties, so that (15) is satisfied with $k_{(D-1,1)} \equiv \tilde{\Phi}_{(D-1,1)}^{3}$, ii) (16) does not vanish so that, (15) is not satisfied. In the latter case the interactions are not consistent. Therefore, we conclude that as far as we deal with the consistent interactions (13) is equivalent to

$$
\Omega_{0} \tilde{\Phi}_{(D, 0)}^{M}+d \tilde{\Phi}_{(D-1,1)}^{M}=0
$$

and it can be generalized to $D$-total form:

$$
\left(\Omega_{0}+d\right) \tilde{\Phi}_{(D)}^{M}=0
$$

which leads to the descent equations

$$
\begin{aligned}
\Omega_{0} \tilde{\Phi}_{(D, 0)}^{M}+d \tilde{\Phi}_{(D-1,1)}^{M} & =0, \\
\Omega_{0} \tilde{\Phi}_{(D-1,1)}^{M}+d \tilde{\Phi}_{(D-2,2)}^{3} & =0, \\
\cdot & \\
\Omega_{0} \tilde{\Phi}_{(1, D-1)}^{M}+d \tilde{\Phi}_{(0, D)}^{M} & =0, \\
\Omega_{0} \tilde{\Phi}_{(0, D)}^{M} & =0 .
\end{aligned}
$$

Obviously some of $\tilde{\Phi}_{(a, b)}^{M}$ can be vanishing. We conclude that the generalized fields method provide the consistent interactions as well as the solution of the descent equations.

Gauge invariance of the interacting theory can be obtained by replacing $S_{0}$ with $S_{0}+S_{\text {int }}$ in (6) on the surface where the ghost fields and the antifields are vanishing.

Spin-1 Gauge Field in D=4: Free theory given by the first order action

$$
\mathcal{L}_{0}=\frac{-1}{2} \int d^{4} x\left(B^{a \mu \nu}\left(\partial_{\mu} A_{\nu}^{a}-\partial_{\nu} A_{\mu}^{a}\right)-\frac{1}{2} B_{\mu \nu}^{a} B^{a \mu \nu}\right),
$$


is invariant under the infinitesimal gauge transformations

$$
\delta_{\Lambda} A_{\mu}^{a}=\partial_{\mu} \Lambda^{a}, \delta_{\Lambda} B_{\mu \nu}^{a}=0 .
$$

The theory is irreducible, so that we need to introduce (in the minimal sector) the ghost field $\eta_{(0,1)}$, and the antifields $A_{(3,-1)}^{\star}, \eta_{(4,-2)}^{\star}$, and $B_{(2,-1)}^{\star}$. Here the star indicates the antifields as well as the Hodge-map.

The generalized fields are

$$
\begin{aligned}
\tilde{A}^{a} & =A_{(1,0)}^{a}+\eta_{(0,1)}^{a}+B_{(2,-1)}^{\star a}, \\
\tilde{B}^{a} & =-A_{(3,-1)}^{\star a}-\eta_{(4,-2)}^{\star a}+B_{(2,0)}^{a} .
\end{aligned}
$$

The proper solution of the master equation is

$$
\begin{aligned}
S_{0} & =\frac{-1}{2} \int d^{4} x\left(\tilde{B}^{a} d \tilde{A}^{a}-\frac{1}{2} \tilde{B}^{a} \tilde{B}^{a}\right)_{(4.0)}, \\
& =-\int d^{4} x\left(\frac{1}{2} B^{a \mu \nu}\left(\partial_{\mu} A_{\nu}^{a}-\partial_{\nu} A_{\mu}^{a}\right)-A_{\mu}^{\star a} \partial^{\mu} \eta^{a}-\frac{1}{4} B_{\mu \nu}^{a} B^{a \mu \nu}\right),
\end{aligned}
$$

from which we read the BRST transformations

$$
\begin{array}{lll}
\Omega_{0} A_{\mu}^{a}=\partial_{\mu} \eta^{a} ; & \Omega_{0} B^{a \mu \nu}=0 ; & \Omega_{0} \eta^{a}=0 ; \\
\Omega_{0} A^{\star a \mu}=\partial_{\nu} B^{a \mu \nu} ; & \Omega_{0} B_{\mu \nu}^{\star a}=\partial_{\mu} A_{\nu}^{a}-\partial_{\nu} A_{\mu}^{a}-B_{\mu \nu}^{a} ; & \Omega_{0} \eta^{a \star}=-\partial_{\mu} A^{\star a \mu} .
\end{array}
$$

The unique candidate for a consistent cubic interaction is the 4-total form

$$
\kappa_{(4)} \equiv \frac{1}{2} f_{a b c} \tilde{B}^{a} \tilde{A}^{b} \tilde{A}^{c} .
$$

One can show that, in fact, (27) provides the solution of the descent equations

$$
\begin{aligned}
\Omega_{0} \kappa_{(4,0)}+d \kappa_{(3,1)} & =0, \\
\Omega_{0} \kappa_{(3,1)}+d \kappa_{(2,2)} & =0, \\
\Omega_{0} \kappa_{(2,2)} & =0, \\
\kappa_{(1,3)}=\kappa_{(0,4)} & =0,
\end{aligned}
$$

if $f_{a b c}$ is totally antisymmetric. Written explicitly

$$
\begin{aligned}
\kappa_{(4,0)} & =f_{a b c}\left(\frac{1}{2} B^{a \mu \nu} A_{\mu}^{b} A_{\nu}^{c}-A^{\star a \mu} A_{\mu}^{b} \eta^{c}+B^{a \mu \nu} \eta^{b} B_{\mu \nu}^{\star c}-\frac{1}{2} \eta^{\star a} \eta^{b} \eta^{c}\right), \\
\kappa_{(3,1)} & =f_{a b c}\left(B^{\mu \nu} A_{\nu}^{b} \eta^{c}+\frac{1}{2} A^{\star a \mu} \eta^{b} \eta^{c}\right) \\
\kappa_{(2,2)} & =-\frac{1}{2} f_{a b c} B^{a \mu \nu} \eta^{b} \eta^{c}
\end{aligned}
$$


If $f_{a b c}$ satisfy the Jacobi identities, one can show that $S_{31}=g_{3} \int \kappa_{(4,0)}$ satisfies

$$
\left(S_{31}, S_{31}\right)=0
$$

Hence, there is no need of adding a quartic interaction with $g_{3}^{2}$ coupling constant. However, one can in principle add a 4 -total form $\tilde{A}^{4}$ with another coupling constant $g_{4}$. For being a consistent interaction $S_{41}$ should be BRST invariant. But one can show that

$$
\Omega_{0} \tilde{A}_{(4,0)}^{4} \neq d K_{(3,1)}
$$

for any $K$. Therefore, we cannot add this interaction term. Observe that after a gauge fixing $B^{\star}=0$ and using the equations of motion related to $B^{\mu \nu}$, Yang-Mills theory follows with the required quartic interaction.

Spin-1 Gauge Field in D=3: The free gauge theory is

$$
\mathcal{A}_{0}=\int A^{a} \wedge d A^{a}
$$

It is invariant under the infinitesimal gauge transformations $\delta_{\Lambda} A^{a}=d \Lambda^{a}$. It is an irreducible theory, so that the generalized field is

$$
\tilde{A}^{a}=A_{\mu(1,0)}^{a}+\eta_{(0,1)}^{a}+A_{\mu(2,-1)}^{\star a}+\eta_{(3,-2)}^{\star a} .
$$

The solution of the master equation

$$
S_{0}=\int \tilde{A}^{a} d \tilde{A}^{a}
$$

leads to the BRST transformations

$$
\begin{gathered}
\Omega_{0} A_{\mu}^{a}=\partial_{\mu} \eta^{a}, \quad \Omega_{0} \eta^{a}=0, \\
\Omega_{0} A^{\star a \mu \nu}=-\epsilon^{\mu \nu \rho} \partial_{\nu} A_{\rho}^{a}, \quad \Omega_{0} \eta^{\star a}=-\partial_{\mu} A^{\star a \mu} .
\end{gathered}
$$

The unique candidate for a consistent interaction is the ghost zero component of the 3 -total form $\xi \equiv \tilde{A}^{3}$. Indeed, one can show that if $f_{a b c}$ are antisymmetric in all of the indices the descent equations

$$
\left(\Omega_{0}+d\right)\left(f_{a b c} \tilde{A}^{a} \tilde{A}^{b} \tilde{A}^{c}\right)_{(3)}=0
$$


are satisfied, where the components are

$$
\begin{aligned}
& \xi_{(3,0)}=-f_{a b c}\left(A^{a} \wedge A^{b} \wedge A^{c}+6 A^{\star a} \wedge A^{b} \wedge \eta^{c}+3 \eta^{\star a} \eta^{b} \eta^{c}\right), \\
& \xi_{(2,1)}=3 f_{a b c}\left(A^{a} \wedge A^{b} \wedge \eta^{c}+A^{\star a} \wedge \eta^{b} \wedge A^{c}\right), \\
& \xi_{(1,2)}=3 f_{a b c} A^{a} \wedge \eta^{b} \wedge \eta^{c} \\
& \xi_{(0,3)}=-f_{a b c} \eta^{a} \wedge \eta^{b} \wedge \eta^{c} .
\end{aligned}
$$

There is no need of any further interaction term because, the total action $S=S_{0}+g_{3} \int \xi_{(3,0)}$ satisfies the master equation if $f_{a b c}$ satisfy the Jacobi identities.

Free BF Theory in $D=2$ : Deal with the free gauge theory given by the Lagrange density

$$
\mathcal{L}_{0}=-\frac{1}{2} \epsilon^{\mu \nu} \Phi^{a}\left(\partial_{\mu} h_{\nu}^{a}-\partial_{\nu} h_{\mu}^{a}\right),
$$

which is invariant under the infinitesimal gauge transformations

$$
\delta h_{\mu}^{a}=\partial_{\mu} \lambda^{a}, \delta \Phi^{a}=0 .
$$

Although a careful treatment of the global modes showed that at some points of the target manifold the theory is reducible[11], we deal with the regions of the target manifold which does not include these points. Hence, the generalized fields are

$$
\begin{gathered}
\tilde{h}^{a}=h_{(1,0)}^{a}+\eta_{(0,1)}^{a}+\Phi_{(2,-1)}^{\star a}, \\
\tilde{\Phi}^{a}=-h_{(1,-1)}^{\star a}-\eta_{(2,-2)}^{\star a}+\Phi_{(0,0)}^{a} .
\end{gathered}
$$

The BV quantized free action

$$
S_{0}=\frac{1}{2} \int \tilde{\Phi}^{a} d \tilde{h}^{a}
$$

leads to the BRST transformations

$$
\begin{array}{ccl}
\Omega_{0} h_{\mu}^{a}=\partial_{\mu} \eta^{a}, & \Omega_{0} \Phi^{a}=0, & \Omega_{0} \eta^{a}=0, \\
\Omega_{0} h^{\star a \mu}=\epsilon^{\mu \nu} \partial_{\nu} \Phi^{a}, & \Omega_{0} \Phi^{\star a}=\epsilon^{\mu \nu} \partial_{\mu} h_{\nu}^{a}, & \Omega_{0} \eta^{\star a}=-\partial_{\mu} h^{\star a \mu} .
\end{array}
$$

There is a unique candidate for a consistent cubic interaction:

$$
S_{31}=g_{3} \int \sigma_{(2,0)} \equiv g_{3} \frac{1}{2} \int d^{2} x f_{a b c}\left(\tilde{\Phi}^{a} \tilde{h}^{b} \tilde{h}^{c}\right)_{(2,0)} .
$$


In fact, one can show that $\sigma_{(D)}$ written in components

$$
\begin{aligned}
\sigma_{(2,0)} & =f_{a b c}\left(\frac{1}{2} \epsilon^{\mu \nu} h_{\mu}^{a} h_{\nu}^{b} \Phi^{c}-h^{\star a \mu} h_{\mu}^{b} \eta^{c}+\Phi^{\star a} \Phi^{b} \eta^{c}-\frac{1}{2} \eta^{\star a} \eta^{b} \eta^{c}\right), \\
\sigma_{(1,1)} & =f_{a b c}\left(h_{\mu}^{a} \eta^{b} \Phi^{c}-\frac{1}{2} h_{\mu}^{\star a} \eta^{b} \eta^{c}\right) \\
\sigma_{(0,2)} & =-\frac{1}{2} f_{a b c} \eta^{a} \eta^{b} \Phi^{c}
\end{aligned}
$$

satisfy the descent equations if $f_{a b c}$ is antisymmetric in all of the indices. Moreover, if they satisfy the Jacobi identities one can show that

$$
\left(S_{1}, S_{1}\right)=0
$$

Although, there is no need of quartic interaction with a coupling constant $g_{3}^{2}$, one can in principle add quartic or higher interaction terms

$$
\sum_{M=4} g_{M} S_{M 1} \equiv \sum_{M=4} g_{M} \int\left(\tilde{\Phi}^{M-2} \tilde{h}^{2}\right)_{(2,0)}
$$

if they satisfy the descent equations (19)-(22). Let us deal with the quartic term

$$
S_{41}=g_{4} \int d^{2} x V_{b c}^{a d}\left(\tilde{\Phi}_{a} \tilde{\Phi}_{d} \tilde{h}^{b} \tilde{h}^{c}\right)_{(2,0)} .
$$

Indeed one can show that

$$
\begin{aligned}
\left(\Omega_{0}+d\right)\left(V_{b c}^{a d} \tilde{\Phi}_{a} \tilde{\Phi}_{d} \tilde{h}^{b} \tilde{h}^{c}\right)_{(2)} & =0 \\
\left(S_{1}, S_{2}\right) & =0 \\
\left(S_{2}, S_{2}\right) & =0
\end{aligned}
$$

if the constants satisfy

$$
\begin{array}{r}
V_{a b}^{c d}=-V_{b a}^{c d}, V_{a b}^{c d}=V_{a b}^{d c}, \\
f_{[a b}^{d} V_{c] d}^{e f}+V_{[a b}^{d f} f_{c] d}^{e}+V_{[a b}^{e d} f_{c] d}{ }^{f}=0, \\
V_{[a b}^{d e} V_{c] d}^{f g}=0,
\end{array}
$$

where [ ] denotes that the indices within them are antisymmetrized.

The higher interactions can also be treated similarly. 
Antisymmetric Tensor Field in D-dimension: The first order free action of the two form field $B=B_{\mu \nu} d x^{\mu} \wedge d x^{\nu}$ in any $\mathrm{D}$ dimensions is

$$
\mathcal{A}_{0}=\int\left(d B^{a} \wedge H^{a}-\frac{1}{2} \hat{H}^{a} \wedge H^{a}\right)
$$

where $H$ is a $D-3$ differential form and $\hat{H}$ is its Hodge dual. (46) is invariant under the gauge transformations

$$
\delta_{\Lambda} B^{a}=d \Lambda^{a}
$$

where $\Lambda^{a}$ is 1-form. The gauge transformations (47) are reducible because, they vanish for $\Lambda^{a}=d \epsilon^{a}$, where $\epsilon^{a}$ is a scalar. Thus, in the minimal sector there are ghost and ghost of ghost fields. The generalized fields are

$$
\begin{aligned}
\tilde{B}_{(2)}^{a} & =B_{(2,0)}^{a}+C_{(1,1)}^{a}+\eta_{(0,2)}^{a}-H_{(3,-1)}^{\star a}, \\
\tilde{H}_{(D-3)}^{a} & =B_{(D-2,-1)}^{\star a}+C_{(D-1,-2)}^{\star a}+\eta_{(D,-3)}^{\star a}+H_{(D-3,0)}^{a},
\end{aligned}
$$

where, now, star indicates also the Hodge map.

Solution of the master equation for the free theory is

$$
S_{0}=\int\left(d \tilde{B}^{a} \tilde{H}^{a}-\frac{1}{2} \hat{H}^{a} \wedge H^{a}\right)_{(D, 0)}
$$

Hence, the BRST transformations are

$$
\begin{array}{llll}
\Omega_{0} B^{a}=d C^{a}, & \Omega_{0} H^{a}=0, & \Omega_{0} C^{a}=d \eta^{a}, & \Omega_{0} \eta^{a}=0, \\
\Omega_{0} B^{\star a}=d H, & \Omega_{0} H^{\star a}=d B-\hat{H}, & \Omega_{0} C^{\star a}=-d B^{\star a}, & \Omega_{0} \eta^{\star a}=d C^{\star a} .
\end{array}
$$

There are four combinations of the generalized fields which are cubic: 1) $\tilde{H}^{3}$ is a $3(D-3)$-total form. For being a Lagrange density the form degree should be $D$, which means that the dimension should be $D=9 / 2$. Hence, it is not permitted.

2) $\tilde{B}^{3}$ is 6 -total form so it is permitted in $D=6$. In 6 -dimension

$$
\tilde{B}_{(6,0)}^{3}=f_{a b c}\left(B^{a} \wedge B^{b} \wedge B^{c}+6 B^{a} \wedge C^{b} \wedge H^{\star c}+2 \eta^{\star a} \wedge H^{\star b} \wedge H^{\star c}\right) .
$$

However, one can easily observe that

$$
\Omega_{0} \tilde{B}_{(6,0)}^{3} \neq d \kappa_{(5,1)}
$$


for any $\kappa_{(5,1)}$. Thus this is excluded.

3) $\tilde{H} \tilde{B}^{2}$ is a $(\mathrm{D}+1)$-total form. Therefore it cannot be a Lagrange density.

4) $\tilde{H}^{2} \tilde{B}$ is $(2 D-4)$-total form thus it is only permitted in $D=4$.

Before dealing with the case 4 let us see if there can be some other consistent interactions which are quartic or higher. There can be

$$
\left(\tilde{H}^{m} \tilde{B}^{n}\right)_{(D, 0)} ; m, n \leq 1, m+n>3,
$$

which is a $(m(D-3)+2 n)$-total form. Thus the dimensions should be

$$
D=\frac{3 m-2 n}{m-1}
$$

if the interactions are consistent. However, one can easily observe that there is not any $n, m$ which lead to an acceptable dimension. Hence we can conclude that the unique candidate for a consistent interaction is the case 4 .

In fact in $D=4, \omega_{(4)} \equiv \tilde{H}^{2} \tilde{B}$ leads to the descent equations

$$
\begin{aligned}
\Omega_{0} \omega_{(4,0)}+d \omega_{(3,1)} & =0, \\
\Omega_{0} \omega_{(3,1)}+d \omega_{(2,2)} & =0, \\
\Omega_{0} \omega_{(2,2)} & =0, \\
\omega_{(1,3)}=\omega_{(0,4)} & =0 .
\end{aligned}
$$

The components written explicitly are

$$
\begin{aligned}
& \omega_{(4,0)}=f_{a b c}\left(H^{a} \wedge H^{b} \wedge B^{c}+2 H^{a} \wedge B^{\star b} \wedge C^{a}-2 H^{a} \wedge C^{\star b} \wedge \eta^{c}+B^{\star a} \wedge B^{\star b} \wedge \eta^{c}\right), \\
& \omega_{(3,1)}=f_{a b c}\left(-H^{a} \wedge H^{b} \wedge C^{c}+2 H^{a} \wedge B^{\star b} \wedge \eta^{c}\right) \\
& \omega_{(2,2)}=f_{a b c} H^{a} \wedge H^{b} \wedge \eta^{c}
\end{aligned}
$$

where $f_{a b c}$ is totally antisymmetric in its indices. Moreover, one can show that if $f_{a b c}$ satisfy the Jacobi identities

$$
S_{31}=g_{3} \int \omega_{(4,0)}
$$

satisfies the master equation:

$$
\left(S_{31}, S_{31}\right)=0 \text {. }
$$

The result which we obtained agree with [6], where the same problem is considered in terms of cohomological techniques. 


\section{References}

[1] T. Kugo and I. Ojima, Prog. Theor. Phys. (Suppl.) 66 (1979) 1.

[2] R. Arnowitt and S. Deser, Nucl. Phys. 49 (1963),

J. Fang and C. Fronsdal, J. Math. Phys. 20 (1979) 2264, F.A. Berends, G.H. Burgers and H. Van Dam, Nucl. Phys. B 260 (1985) 295 ,

A.K.H. Bengtsson, Phys. Rev. D 32 (1985) 2031.

[3] I.A.Batalin and G.A.Vilkovisky, Phys. Lett. B 102 (1981) 27; Phys. Rev. D 28 (1983) 2567.

[4] G. Barnich and M. Henneaux, Phys. Lett. B 311 (1993) 123.

[5] G. Barnich, M. Henneaux and R. Tatar, Consistent Interactions Between Gauge Fields and Local BRST Cohomology: The Example of Yang-Mills Models, ULB-PMIF/93-04, 1993.

[6] M. Henneaux, Uniqueness of the Freedman-Townsend Interaction Vertex for Two-Form Gauge Fields, hep-th/9511145, 1995.

[7] Ö.F. Dayi, Mod. Phys. Lett. A 8 (1993) 811; ibid. 2087.

[8] Ö.F. Dayi, Inter. J. Mod. Phys. A 11 (1996) 1.

[9] N. Ikeda and K.I. Izawa, Prog. Theor. Phys. 89 (1993) 1077; ibid. 90 (1993) 237.

[10] N. Dragon, BRS Symmetry and Cohomology, ITP-UH-3/96, hepth/9602163.

[11] P. Schaller and T. Strobl, Mod. Phys. Lett. A 9 (1994) 3129. 